\title{
Building Compact Recognizer with Recognition Rate Maintained for On-line Handwritten Japanese Text Recognition
}

\author{
Jinfeng $\mathrm{GAO}^{\mathrm{a}, \mathrm{b}}$, Bilan ZHU ${ }^{\mathrm{a}}$, Masaki NAKAGAWA ${ }^{\mathrm{a}}$ \\ ${ }^{a}$ Department of Computer and Information Sciences, Tokyo University of Agriculture and Technology, Tokyo, Japan \\ ${ }^{b}$ Department of Computer and Information Sciences, Huanghuai University, Zhumadian, China
}

\begin{abstract}
The paper presents complexity reduction of an on-line handwritten Japanese text recognition system by selecting an optimal off-line recognizer in combination with an on-line recognizer, geometric context evaluation, and linguistic context evaluation. The result is that a surprisingly simple off-line recognizer, which is weak on its own, produces nearly the best recognition rate in combination with other evaluation factors in remarkably small space-and-time complexity. Generally, lower dimensions with fewer principal components produce a smaller set of prototypes, which reduces memory-cost and time-cost. This degrades the recognition rate, however, so we need to reach a compromise. In an evaluation function with the above-mentioned multiple factors combined, the configuration of only 50 dimensions with as few as 5 principal components for the off-line recognizer keeps almost the best accuracy $98.23 \%$ (the best accuracy $98.34 \%$ ) for text recognition while it reduces the total memory-cost to $1 / 3$ (from $99.4 \mathrm{MB}$ down to 32 $\mathrm{MB}$ ) and the average time-cost of character recognition for text recognition to $4 / 5$ (from $0.1672 \mathrm{~ms}$ to $0.1349 \mathrm{~ms}$ per character) compared with the traditional off-line recognizer with 160 dimensions and 50 principal components.
\end{abstract}

Keywords: on-line recognition, integrated recognition model, combined recognizer, complexity reduction, linguistic context, geometric context

\section{INTRODUCTION}

With the development of pen-based or touch-based mobile terminals, handwriting recognition systems running 3 on such mobile terminals need to be developed. The relatively small RAM of mobile terminals requires handwriting 4 recognition systems to be as small as possible while keeping accuracy high. This paper focuses on constructing an on5 line handwritten Japanese text recognition system running on such terminals. Japanese uses thousands of ideographic characters of Chinese origin, two sets of phonetic characters, the Roman alphabet, numerals, and symbols, so the method is general and applicable to other languages, especially those that use many sets of characters.

Handwritten character recognition mainly includes two types of methods: on-line and off-line recognition. The on-line method is robust to stroke connection and deformation but not to stroke order variation. On the other hand, the

Email addresses: gaojinfeng19790213@yahoo.co.jp (Jinfeng GAO), zhubilan@cc.tuat.ac.jp (Bilan ZHU), nakagawa@cc. tuat.ac.jp (Masaki NAKAGAWA)

Preprint submitted to Pattern Recognition Letter

August 5, 2013 
off-line method is robust to stroke order variation and duplicated strokes but not to stroke connection and deformation.

To overcome the disadvantage of the on-line method, off-line recognition is combined with on-line recognition to form a combined recognizer [1].

For Chinese and Japanese character recognition, MQDF (Modified Quadratic Discriminant Function) proposed by Kimura et al. [2] has been often used, but its performance is dependent on two parameters: the size of feature dimensions and the number of principal components. Using the different number of dimensions and principal components, we can obtain a variety of off-line recognizers with different performances, memory-costs, and recognition speeds. The required memory ranges from a few $\mathrm{MB}$ to about $100 \mathrm{MB}$, time-cost ranges from a few ms to tens of ms, and so on. Therefore, there are many choices for off-line recognizers in handwritten character recognition systems.

For on-line handwritten text recognition, an entire system includes not only an off-line character recognizer but also an on-line recognizer and other components for linguistic context, geometric context, and so on [3, 4, 5]. Therefore, we must design the off-line character recognizer so that it performs as well as possible with other components while satisfying memory and speed constraints. This is true for each of the other components, but the off-line recognizer based on MQDF is large and time consuming, so its design makes up a large portion in the entire system design. Being not only important but also scalable by adjusting parameters, the off-line recognizer can be designed to optimize the system.

In our previous work, we reported an example of such a system composed of off-line and on-line recognizers with bi-gram context [6]. In this paper, by using improved components, we consider the effect of scaling off-line recognizer in the entire text recognition system more thoroughly and report even higher recognition rate while decreasing the dimension size and the number of principal components even further. The rest of this paper is organized as follows: Section 2 describes an overview of the text recognition system. Sections 3 to 5 explain the process of constructing the combined recognizer; Section 6 presents the evaluation function for optimal path search. Section 7 reports experimental results and analysis. Finally, we make the concluding remarks.

\section{OVERALL SYSTEM AND EVALUATION DATABASE}

\subsection{Overall System}

Figure 1(a) shows the flow chart of our on-line handwritten Japanese text recognition system. We can see that the process mainly consists of three modules: pre-processing, generation of a candidate segmentation and recognition lattice (csr-lattice), and optimal path search. The pre-processing part is used to normalize and smooth each stroke (a time-sequence of pen-tip coordinates from pen-down to pen-up) of handwritten text. In the stage of constructing a csr-lattice, a stroke sequence for a text string is segmented into blocks (primitive segments) in accordance with the geometric features such as off-strokes (a vector from pen-up to the next pen-down) and overlap of bounding boxes of adjacent strokes. One or more consecutive primitive segments form a candidate character pattern marked by solid line boxes as shown in Figure 1(b). Concatenation of consequent primitive segments to form a candidate 


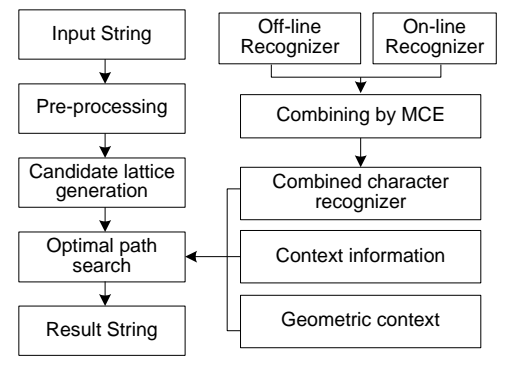

(a) Flow chart of handwritten text recognition

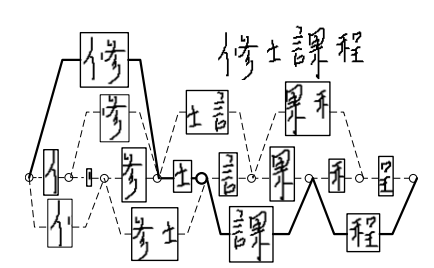

(b) Lattice of candidate patterns

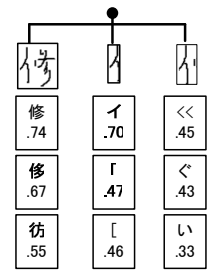

(c) Lattice of candidate character

Figure 1: Flow chart of handwritten text recognition, and Candidate segmentation and recognition lattice.

character pattern is limited by a clear segmentation point judged by the geometric features or their total length. Then, each candidate character pattern is recognized, and several pairs of a character recognition candidate and its score are listed with the candidate character pattern. A csr-lattice represents multiple ways of segmentation and multiple ways of recognition in the form of a lattice. Figure 1(b) shows an example where each node represents a candidate character pattern and each arc represents segmentation. Each node stores multiple pairs of a character recognition candidate and its score by a character recognizer. For instance, three starting character patterns shown leftmost in Figure 1(b) have their recognition candidates and scores shown in Figure 1(c). Those for other nodes in Figure 1 (b) are omitted for simplicity. Each path in a csr-lattice represents a sequence of candidate character patterns and character categories recognized for them. Handwritten text recognition integrates character segmentation and recognition because character patterns cannot be reliably segmented without being recognized due to the irregularity of character size and spacing [4]. It is made by optimal path search in a csr-lattice, which will be described in Section 6.

\subsection{Evaluation Database}

To evaluate performance of recognizers constructed in different stages, we experimented with the TUAT HANDS database, Kuchibue and Nakayosi databases of on-line handwritten Japanese characters [7], and Kondate database of on-line text. The Kuchibue database contains on-line handwritten character patterns of 120 writers, 11,962 patterns per writer covering 3,356 character classes. The 11,962 patterns per writer include two parts: the sentential part consisting of 10,154 characters, often used for text recognition testing and 1,808 single characters that appear less often. The Nakayosi database contains the samples of 163 writers, 10,403 patterns covering 4,438 character classes per writer. Therefore, Kuchibue in total contains $11,962 \times 120=1,435,440$ character patterns and Nakayosi $10,403 \times 163=1,695,689$ patterns.

For Kuchibue and Nakayosi, each character pattern was written in each writing box, while for Kondate, character patterns were written freely without any writing grid and even without line drawings by 100 writers. 


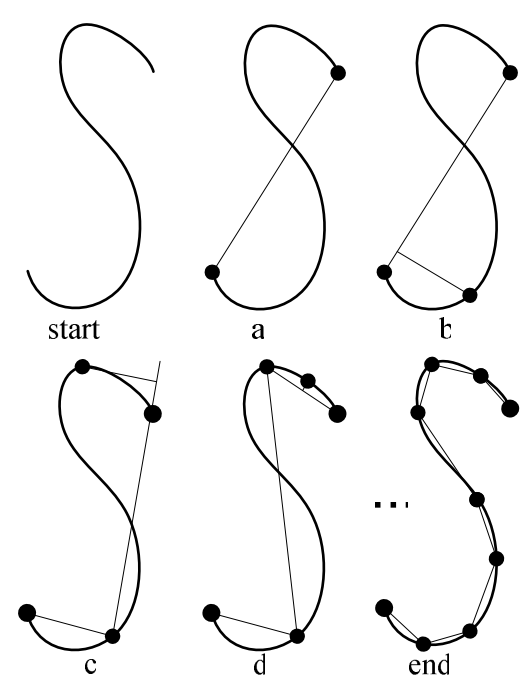

(a) Feature points extraction

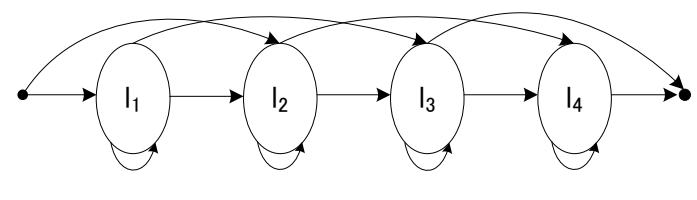

(b) A linear MRF chain

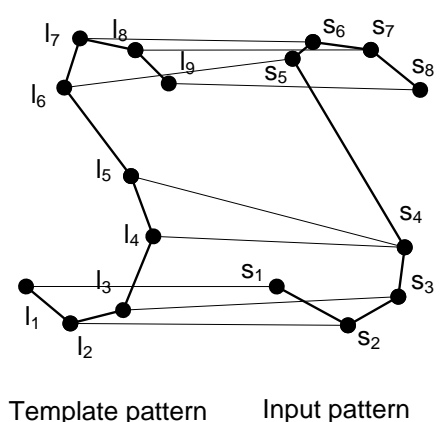

(c) Mapping from $S$ to $L$

Figure 2: (a) Feature points extraction, (b) MRF model used in this paper and (c) Mapping from $S$ to $L$.

\section{ON-LINE CHARACTER RECOGNIZER AND EVALUATION}

The character recognition in text recognition is based on a combining an on-line and an off-line recognizer. This section describes the on-line recognizer briefly and its performance. Although the Hidden Markov Model (HMM) is a directed graph, it just matches pen-points (feature points) of an input pattern with states for each character class probabilistically [8]. However, the Markov Random Field (MRF) can effectively integrate the information between neighboring pen-points such as binary and triple pen-points and information from single pen-points to improve robustness to classification [9][10]. We have shown that MRF provides better recognition than HMM for Japanese character recognition in our previous work [11].

\subsection{Feature Points Extraction}

In on-line handwritten character recognition, it is more effective and efficient to use extracted feature points instead of all the pen-tip points to express character patterns. We normalize an input character pattern linearly to a standard size while preserving the horizontal-vertical ratio and extract its feature points. For each stroke, first, the start and end points are picked up as feature points; then, the point farthest from the straight line through adjacent feature points is selected as a feature point while the distance is greater than a threshold. This process continues recursively until no more feature points are selected [12], which is described by Figure 2(a). 


\subsection{MRF Model}

We represent feature points from an input pattern as sites $S=\left\{s_{1}, s_{2}, \ldots, s_{I}\right\}$ and states of a character class $\mathrm{C}$ as labels $L=\left\{l_{1}, l_{2}, \ldots, l_{J}\right\}$. We assume a linear-chain MRF (Markov Random Field) for each character class as shown in Figure 2 (b), where each label has a state and each state has three transitions. Moreover, we represent the mapping from $S$ to $L$ for character recognition as $F=\left\{s_{1}=l_{i}, s_{2}=l_{j}, \ldots, s_{I}=l_{k}\right\}$, which is called as a configuration as shown in Figure 2(c). Since there are more than one configuration F, making matching under all Fs is intractable. Therefore, we just consider the best figuration $F_{\text {best }}$ which is obtained by the Viterbi algorithm [13].

For simplicity, we consider only single-site cliques $c l_{1}=\left\{s_{i}\right\}$ and pair-site cliques $c l_{2}=\left\{\left(s_{i-1}, s_{i}\right)\right\}$. The feature vector extracted from feature points of an input pattern $\mathrm{X}$ is considered as the observation set $O$. $O$ is composed of a unary feature vector extracted from $s_{i}$ site noted as $O_{s_{i}}$ and a binary feature vector extracted from the pair-site $s_{i-1}$ and $s_{i}$ noted as $O_{s_{i-1} s_{i}}$. The energy function of MRF for an input pattern against the C-th class is given by Eq. (1), where $O_{s_{i}}$ consists of coordinates $x$ and $y$, and $O_{s_{i-1} s_{i}}$ consists of difference of $x$ between sites $s_{i-1}$ and $s_{i}$ and that of y. $P\left(O_{s_{i}} \mid l_{s_{i}}, C\right)$ and $P\left(O_{s_{i-1} s_{i}} \mid l_{s_{i-1}} l_{s_{i}}, C\right)$ are evaluated by Gaussian functions. $P\left(l_{s_{i}} \mid l_{s_{i-1}}, C\right)$ is calculated by Eq. (2). The detail of the energy function of MRF is described in our previous work [11].

As for the MRF model for each character class, it consists of means and variances of unary features extracted from single-site sites, means and variances of binary features extracted from adjacent feature points, and the transition probability between adjacent feature points, based on Eq. (1).

$$
\begin{aligned}
E\left(O, F_{\text {best }} \mid C\right) & =E\left(F_{\text {best }} \mid C\right)+E\left(O \mid F_{\text {best }}, C\right) \\
& =\sum_{i=1}^{I}\left[-\log P\left(O_{s_{i}} \mid l_{s_{i}}, C\right)-\log P\left(O_{s_{i-1} s_{i}} \mid l_{s_{i-1}} l_{s_{i}}, C\right)-\log P\left(l_{s_{i}} \mid l_{s_{i-1}}, C\right)\right]
\end{aligned}
$$

$$
P\left(l_{s_{i}} \mid l_{s_{i-1}}, C\right)=\frac{\text { Number of transitions from } l_{s_{i-1}} \text { to } l_{s_{i}}}{\text { Number of sites assigned to } l_{s_{i-1}}}
$$

\subsection{Evaluation of On-line Recognizer}

We use Eq. (1) to obtain evaluation score between an input pattern and each candidate character class. It is addressed that the evaluation score does not meet the value range is from zero to one, but meets the characteristic of similarity: the greater the value, the greater reliability of the candidate character class. Therefore, the candidate character class with the largest evaluation score is considered as the recognition result. In our system, the evaluation score ranges in [0, 2000], mainly falls in [0,600]. After the Nakayosi database is used to train the MRF model and the Kuchibue database is used for testing, the accuracy of the on-line recognizer is $92.77 \%$, the memory-cost is 12 MB and average time-cost of per character is about $21 \mathrm{~ms}$. The experiment is done on a computer with Intel Core 2 Duo CPU E8500 of 3.16 GHz and 4-GB RAM. 


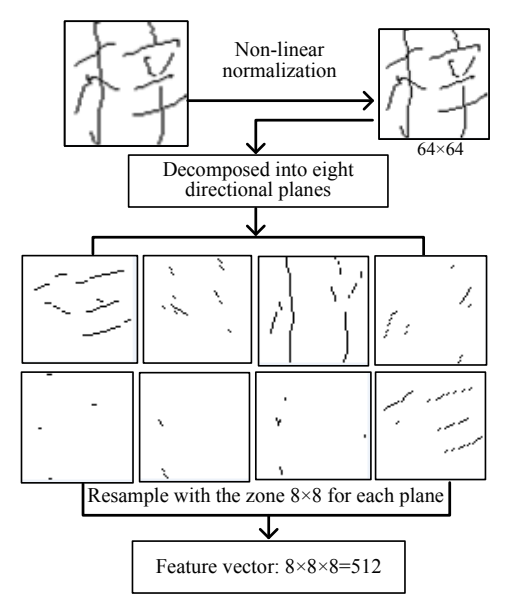

Figure 3: Feature vector extraction.

\section{OFF-LINE CHARACTER RECOGNIZER AND EVALUATION}

In this section, we describe feature extraction, an algorithm for feature reduction, and a discriminant function for the off-line recognizer.

\subsection{Feature Extraction}

For the off-line recognizer, we extract directional features: histograms of normalized stroke direction [14] from an on-line character pattern (a sequence of stroke coordinates). For coordinate normalization we apply pseudo 2D bi-moment normalization (P2DBMN) [15]. The local stroke direction is decomposed into 8 direction planes, and $8 \times 8$ regions are extracted from each direction plane by the Gaussian blurring, so the dimensionality of feature vectors is 512. This process is shown in Figure 3. To improve the Gaussianity of feature distribution, each value of the 512 features is transformed by the Box-Cox transformation [16] (also called variable transformation).

\subsection{Fisher Linear Discriminate Analysis}

Fisher discriminant analysis (FDA) is well known and common in pattern recognition and feature reduction. Suppose there are $C$ known character classes $\omega_{1}, \omega_{2}, \ldots \omega_{C}$ and the ith class with $N_{i}$ training samples, in total $N$ training samples. $X=\left\{x_{j}^{i}\right\}\left(j=1,2, \ldots C, i=1,2, \ldots N_{j}\right)$ is a set of samples with $n$-dimensions where $N_{j}$ is the number of samples of $j$ th class. The mean vectors of each class and all classes can be expressed as $\bar{X}_{j}=\left(1 / N_{j}\right) \sum_{i=1}^{N_{j}} x_{j}^{i}$ and $\bar{X}=(1 / N) \sum_{j=1}^{C} \sum_{i=1}^{N_{j}} x_{j}^{i}$, respectively. Let the between-class and within-class scatter matrices be defined as:

$$
S_{b}=\sum_{j=1}^{C} N_{j}\left(\bar{X}_{j}-\bar{X}\right)\left(\bar{X}_{j}-\bar{X}\right)^{T}
$$

$$
S_{w}=\sum_{j=1}^{C} \sum_{i=1}^{N_{j}}\left(x_{j}^{i}-\bar{x}_{j}\right)\left(x_{j}^{i}-\bar{x}_{j}\right)^{T}
$$


The process to obtain the transformation matrix is maximizing the following quotient, called the Fisher discriminant criterion $[17,18]$.

$$
w_{\text {opt }}=\operatorname{argmax}_{w}\left|\frac{w^{T} S_{b} w}{w^{T} S_{w} w}\right|=\left[w_{1}, w_{2}, \ldots, w_{m}\right]
$$

where $\left\{w_{i} \mid i=1,2, \ldots, m\right\}$ are $m n$-dimensional eigenvectors of $S_{w}^{-1} S_{b}$ corresponding to the $m$ largest eigenvalues. $w_{\text {opt }}$ is a $n \times m$ matrix composed of the $m n$-dimensions eigenvectors. By the transformation matrix $w_{\text {opt }}$, we can reduce the feature dimensions from $n$-dimensions to $m$-dimensions.

\section{3. $M Q D F 2$}

We employ MQDF since it has been most often employed for Chinese and Japanese character recognition. It is superior to Quadratic Discriminant Function (QDF) in recognition rate, speed and memory size. Historically, the Nearest Neighbor method was first employed for OCR(Optical Character Recognition) but replaced by MQDF. Neural Network has been tested but could not cope with thousands of categories. Discriminative classifiers such as support vector machine (SVM) are also candidates, but statistical classifiers can generalize better than discriminative ones when the amount of sample patterns is limited. In addition, parametric statistical classifiers are resistant to outliers (non-characters), whereas discriminative classifiers are susceptible to outliers because their decision regions tend to open. Considering that the amount of training patterns is limited and the off-line recognizer in this paper is employed for text recognition, in which there are many non-character candidate patterns during generation of csr-lattice, we employ the most established statistical classifier: MQDF rather than discriminative classifiers such as SVM. Kimura et al. proposed two types of modified quadratic discriminant functions (MQDF1 and MQDF2) which are less sensitive to the estimation error of the covariance to QDF in [2]. MQDF1 employs a kind of Bayesian estimate of the covariance matrix instead of the maximum likelihood estimate, while MQDF2 is a smoothed version of QDF by replacing the minor eigenvalues with a larger constant.

For an input character pattern $X=\left(x_{1}, \ldots, x_{n}\right)^{T}$, the MQDF2 for class $\omega_{i}, i=1,2, \ldots, N$, is obtained as

$$
g_{2}\left(X, w_{i}\right)=\sum_{j=1}^{k} \frac{1}{\lambda_{i j}}\left[\left(X-u_{i}\right)^{T} \varphi_{i j}\right]^{2}+\frac{1}{\delta_{i}} D_{c}(X)+\sum_{j=1}^{k} \log \lambda_{i j}+(n-k) \log \delta_{i}
$$

45

$$
\begin{aligned}
& D_{c}(X)=\left\|X-u_{i}\right\|^{2}-\sum_{j=1}^{k}\left[\left(X-u_{i}\right)^{T} \varphi_{i j}\right]^{2} \\
& \delta_{i}=\frac{\operatorname{tr}\left(\sum_{i}\right)-\sum_{j=1}^{k} \lambda_{i j}}{n-k}=\frac{1}{n-k} \sum_{j=k+1}^{n} \lambda_{i j}
\end{aligned}
$$


Then, the size of the off-line prototype dictionary $S$ is depended on the data type of the parameters $u_{i}, \lambda_{i j}, \varphi_{i j}, \delta_{i}$ which are labeled as $T_{u}, T_{\lambda}, T_{\varphi}, T_{\delta}$, respectively. In our system, $T_{u}, T_{\lambda}, T_{\varphi}$ are integers with 16 bits; $T_{\delta}$ is a long integer with 32 bits. We can have the total size of the prototype dictionary given by Eq. (9) and $\mathrm{N}$ is the number of the character categories. In our system, $\mathrm{N}$ is 4438.

$$
S=N \times\left\{\left(n \times T_{u}+k \times T_{\varphi}\right)+k \times T_{\lambda}+T_{\delta}\right\}
$$

\subsection{Evaluation of Off-line Recognizer}

To evaluate performance of the off-line recognizer, we use the Nakayosi database as training data and the Kuchibue database as the testing data. The evaluation is implemented on Intel Core 2 Duo CPU E8500 of 3.16 GHz and a 4-GB

RAM computer.

Table 1: Performance evaluation of different off-line character recognizers

(a) Accuracies of different off-line recognizers (\%)

\begin{tabular}{|c|c|c|c|c|c|c|c|c|c|c|c|c|}
\hline \multicolumn{8}{|c|}{ Recognition accuracy in CLD } & \multicolumn{5}{|c|}{ Recognition accuracy in SLD } \\
\hline $\mathrm{k} \backslash \mathrm{n}$ & 60 & 80 & 100 & 120 & 140 & 160 & 180 & $\mathrm{k} \backslash \mathrm{n}$ & 20 & 30 & 40 & 50 \\
\hline 10 & 87.4 & 88.2 & 88.6 & 88.8 & 89.0 & 89.1 & 89.1 & 2 & 76.8 & 81.6 & 83.4 & 84.5 \\
\hline 20 & 87.5 & 88.3 & 88.9 & 89.2 & 89.5 & 89.7 & 89.9 & 5 & 77.8 & 83.0 & 85.1 & 86.2 \\
\hline 30 & 87.7 & 88.5 & 88.9 & 89.2 & 89.5 & 89.8 & 90.0 & 10 & 78.0 & 83.4 & 85.5 & 86.7 \\
\hline 40 & 88.1 & 88.8 & 89.1 & 89.4 & 89.7 & 89.9 & 90.1 & 13 & 78.6 & 83.5 & 85.6 & 86.8 \\
\hline 50 & 88.6 & 89.1 & 89.4 & 89.7 & 90.0 & 90.1 & 90.3 & 16 & 79.7 & 83.6 & 85.6 & 86.9 \\
\hline 60 & 88.2 & 89.5 & 89.9 & 90.1 & 90.4 & 90.5 & 90.7 & 20 & 81.4 & 84.1 & 85.8 & 86.8 \\
\hline
\end{tabular}

(b) Memory-costs of different off-line character recognizers (MB)

\begin{tabular}{|c|c|c|c|c|c|c|c|c|c|c|c|c|}
\hline \multicolumn{8}{|c|}{ Memory-cost (MB) in CLD } & \multicolumn{5}{|c|}{ Memory-cost (MB) in SLD } \\
\hline $\mathrm{k} \backslash \mathrm{n}$ & 60 & 80 & 100 & 120 & 140 & 160 & 180 & $\mathrm{k} \backslash \mathrm{n}$ & 20 & 30 & 40 & 50 \\
\hline 10 & 5.8 & 7.6 & 9.5 & 11.3 & 13.2 & 15 & 16.9 & 2 & 0.6 & 0.8 & 1.1 & 1.3 \\
\hline 20 & 11.0 & 14.5 & 18.1 & 21.6 & 25.2 & 28.7 & 32.3 & 5 & 1.1 & 1.6 & 2.1 & 2.6 \\
\hline 30 & 16.2 & 21.5 & 26.7 & 32.0 & 37.2 & 42.5 & 47.9 & 10 & 2.0 & 3.0 & 3.9 & 4.8 \\
\hline 40 & 21.5 & 28.4 & 35.3 & 42.3 & 49.2 & 56.2 & 63.1 & 13 & 2.6 & 3.8 & 5.0 & 6.2 \\
\hline 50 & 26.7 & 35.3 & 44.0 & 52.6 & 61.3 & 69.9 & 78.5 & 16 & 3.2 & 4.6 & 6.0 & 7.5 \\
\hline 60 & 32.0 & 42.3 & 52.6 & 62.9 & 73.3 & 83.6 & 93.9 & 20 & 3.9 & 5.7 & 7.5 & 9.2 \\
\hline
\end{tabular}

(c) Average time-costs per characters of different off-line recognizers(ms)

\begin{tabular}{|c|c|c|c|c|c|c|c|c|c|c|c|c|}
\hline \multicolumn{8}{|c|}{ Average time-cost (ms) in CLD } & \multicolumn{5}{|c|}{ Average time-cost (ms) in SLD } \\
\hline $\mathrm{k} \backslash \mathrm{n}$ & 60 & 80 & 100 & 120 & 140 & 160 & 180 & $\mathrm{k} \backslash \mathrm{n}$ & 20 & 30 & 40 & 50 \\
\hline 10 & 0.246 & 0.301 & 0.364 & 0.392 & 0.431 & 0.481 & 0.521 & 2 & 0.115 & 0.136 & 0.158 & 0.180 \\
\hline 20 & 0.311 & 0.393 & 0.441 & 0.525 & 0.559 & 0.676 & 0.703 & 5 & 0.121 & 0.146 & 0.172 & 0.192 \\
\hline 30 & 0.387 & 0.452 & 0.531 & 0.638 & 0.731 & 0.820 & 0.840 & 10 & 0.139 & 0.165 & 0.194 & 0.220 \\
\hline 40 & 0.428 & 0.548 & 0.653 & 0.790 & 0.862 & 0.940 & 1.050 & 13 & 0.141 & 0.174 & 0.204 & 0.238 \\
\hline 50 & 0.516 & 0.675 & 0.758 & 0.898 & 0.990 & 1.125 & 1.343 & 16 & 0.150 & 0.185 & 0.217 & 0.245 \\
\hline 60 & 0.549 & 0.714 & 0.815 & 0.943 & 1.115 & 1.226 & 1.590 & 20 & 0.168 & 0.199 & 0.235 & 0.273 \\
\hline
\end{tabular}

In the process of recognizer training, we extract 512-dimensional features and then reduce them to lower dimensions by FDA. So far, reduction to the range from 60 to 180 has been common. We call this group common lower dimensions (CLD). On the other hand, we consider much lower dimensions from 20 to 50 . We call them super lower 
dimensions (SLD). In CLD, we consider various dimensions from 60 to 180 with the step 20 and in SLD, we consider variations from 20 to 50 with the step 10. For each dimension in CLD, we take a different number of principal components from 10 to 60 with the step 10 so that we produce 42 different off-line recognizers. Similarly, for SLD we take a different number of principal components as $2,5,10,13,16$, and 20, so that we obtain 24 off-line recognizers. We measure the accuracy, memory-cost, and average time-cost per character for the 66 different off-line recognizers (42 in CLD and 24 in SLD) as shown in Table 1, respectively.

From the above table, the variation of accuracy among different recognizers is within $23.9 \%$ (from $76.8 \%$ to 90.7\%); the memory-cost ranges from 0.6 MB to 93.9 MB and the time-cost ranges from $0.115 \mathrm{~ms}$ to $1.590 \mathrm{~ms}$.

\section{COMBINED RECOGNIZER AND EVALUATION}

This section describes the method of combining the on-line recognizer (described in Section 3) and the off-line recognizer (described in Section 4). We firstly apply coarse classification with the memory-cost about $10 \mathrm{MB}$ and then apply the on-line and off-line recognizers to obtain the combined candidates by sum rule [19].

\subsection{Combination of on-line and off-line recognizers}

The two recognizers are combined as follows:

A pattern $s=\left(s_{1}, \cdots, s_{m}\right)^{T}$ is recognized as a character class $c_{i}$ by the on-line and off-line recognizers with their evaluation scores $f_{\text {on }}^{c_{i}}$ and $f_{\text {off }}^{c_{i}}$, respectively. Then, the confidence of the combined recognizer $f_{\text {com }}^{c_{i}}$ by the sum rule with class-independent linear combining parameters is given by Eq. (10).

$$
f_{\text {com }}^{c_{i}}=\lambda_{1} f_{o n}^{c_{i}}+\lambda_{2} f_{o f f}^{c_{i}}
$$

We use the Minimum Classification Error (MCE) criterion [20] to optimize the parameters. On each training pattern, a loss function is computed to approximate the classification error and the empirical loss is minimized to optimize the combining parameters on training patterns. The misclassification measure of a pattern from the class $c_{i}$ is given by Eq. (11), where $f_{c o m}^{r}$ is the evaluation of the closest rival class. The misclassification measure is transformed to give the loss as given by Eq. (12).

$$
\begin{gathered}
u_{c_{i}}=f_{\text {com }}^{r}-f_{\text {com }}^{c_{i}}, \\
l s_{c_{i}}(s)=l s_{c_{i}}\left(u_{c_{i}}\right)=\frac{1}{1+\exp \left(-\xi u_{c_{i}}\right)} .
\end{gathered}
$$

On all the training patterns $\left\{\left(s^{n}, c^{n}\right) \mid n=1,2, \cdots, N\right\}$, the empirical loss is computed by Eq. (13) where $c^{n}$ is the class label of pattern $s^{n}, I(\cdot)$ is an indicator function and $M$ denotes the number of character classes. We minimize $f_{L}$ by stochastic gradient descent to obtain the optimal parameters. The value of $\xi$ should be inversely proportional to the magnitude of mean value of $u_{c_{i}}$ on training samples based on initial parameters $\lambda_{1}$ and $\lambda_{2}$. 


$$
f_{L}=\frac{1}{N} \times \sum_{n=1}^{N} \sum_{i=1}^{M} l s_{i}\left(s^{n}\right) \times I\left(c^{n} \in i\right) .
$$

\subsection{Evaluation of Combined Recognizer}

We combine the on-line recognizer introduced in Section 3 with each of 66 different off-line recognizers described in the previous section to generate 66 different combined recognizers by the combining approach shown in Section 5.1. Using Nakayosi as the training data and Kuchibue as the testing data, we obtained the accuracy and average time-cost per character of 66 different combined recognizers shown in Table 2 (a) and (b), respectively. We use the pair of parameters for the off-line recognizer to mark the combined recognizer in Table 2, because only the off-line recognizer is variable during the combination of on-line and off-line recognizers.

Table 2: Performance evaluation of different combined character recognizers

(a) Accuracies of different combined recognizers (\%)

\begin{tabular}{|c|c|c|c|c|c|c|c|c|c|c|c|c|}
\hline \multicolumn{8}{|c|}{ Recognition accuracy $(\%)$ in CLD } & \multicolumn{5}{|c|}{ Recognition accuracy $(\%)$ in SLD } \\
\hline $\mathrm{k} \backslash \mathrm{n}$ & 60 & 80 & 100 & 120 & 140 & 160 & 180 & $\mathrm{k} \backslash \mathrm{n}$ & 20 & 30 & 40 & 50 \\
\hline 10 & 95.21 & 95.32 & 95.43 & 95.45 & 95.48 & 95.45 & 95.46 & 2 & 91.10 & 92.40 & 93.22 & 93.60 \\
\hline 20 & 95.30 & 95.55 & 95.62 & 95.72 & 95.70 & 95.72 & 95.70 & 5 & 92.21 & 93.50 & 94.20 & 94.53 \\
\hline 30 & 95.17 & 95.49 & 95.63 & 95.70 & 95.72 & 95.72 & 95.73 & 10 & 92.33 & 93.93 & 94.66 & 95.00 \\
\hline 40 & 94.80 & 95.41 & 95.62 & 95.64 & 95.70 & 95.69 & 95.74 & 13 & 91.95 & 93.89 & 94.73 & 95.08 \\
\hline 50 & 93.80 & 95.13 & 95.49 & 95.57 & 95.60 & 95.61 & 95.64 & 16 & 91.60 & 93.74 & 94.64 & 95.07 \\
\hline 60 & 92.97 & 94.38 & 95.14 & 95.40 & 95.46 & 95.48 & 95.44 & 20 & 91.53 & 93.46 & 94.62 & 95.13 \\
\hline
\end{tabular}

(b) Average time-cost per character of different combined recognizer (ms)

\begin{tabular}{|c|c|c|c|c|c|c|c|c|c|c|c|c|}
\hline \multicolumn{8}{|c|}{ Average Time-cost (ms) in CLD } & \multicolumn{5}{|c|}{ Average Time-cost (ms) in SLD } \\
\hline $\mathrm{k} \backslash \mathrm{n}$ & 60 & 80 & 100 & 120 & 140 & 160 & 180 & $\mathrm{k} \backslash \mathrm{n}$ & 20 & 30 & 40 & 50 \\
\hline 10 & 0.1298 & 0.1380 & 0.1329 & 0.1694 & 0.1613 & 0.1675 & 0.1425 & 2 & 0.0972 & 0.1117 & 0.1314 & 0.1207 \\
\hline 20 & 0.1342 & 0.1404 & 0.1372 & 0.1546 & 0.1595 & 0.1610 & 0.1484 & 5 & 0.1267 & 0.1299 & 0.1149 & 0.1349 \\
\hline 30 & 0.1374 & 0.1424 & 0.1409 & 0.1636 & 0.2353 & 0.1728 & 0.1532 & 10 & 0.1052 & 0.1139 & 0.1159 & 0.1208 \\
\hline 40 & 0.1401 & 0.1422 & 0.1436 & 0.1641 & 0.1659 & 0.1721 & 0.1584 & 13 & 0.1055 & 0.1163 & 0.1176 & 0.1565 \\
\hline 50 & 0.1419 & 0.1437 & 0.1438 & 0.1786 & 0.1780 & 0.1622 & 0.1627 & 16 & 0.1053 & 0.1151 & 0.1155 & 0.1229 \\
\hline 60 & 0.1447 & 0.1445 & 0.1459 & 0.1660 & 0.1723 & 0.1773 & 0.1672 & 20 & 0.1215 & 0.1317 & 0.1258 & 0.1265 \\
\hline
\end{tabular}

In Table 2 (a), the accuracy among different combined recognizers varies from $91.10 \%$ to $95.74 \%$, within $14.7 \%$; Moreover, in Table 1 (a) and Table 2 (a), we can see that the accuracy is 5 points higher $(95.74 \%$ when $\mathrm{k}=40$ and $\mathrm{n}=180$ in Table 2 (a)) than that of the single off-line recognizer in the best conditions $(90.7 \%$ when $\mathrm{k}=60$ and $\mathrm{n}=180$ in Table 1 (a)). In Table 2 (b), the time-cost of recognition per character is from 0.0972 to 0.1786 ms, the maximum difference is about $0.0814 \mathrm{~ms}$; moreover, in Table 1 (c) and Table 2 (b), the time-cost is decreased from 1.59 to 0.1786 ms after combination, maximally.

The memory-costs of all combined recognizer are summations among the memory-costs for the coarse classification (10 MB), the on-line recognizer (12 MB) and the corresponding off-line recognizer shown in Table 1 (b). Therefore, the total memory-cost is $22 \mathrm{MB}(10 \mathrm{MB}+12 \mathrm{MB})$ plus memory-cost of the corresponding off-line rec- 
ognizer. The evaluation experiment was also implemented on the same hardware environment as that in off-line recognizer evaluation.

\section{PATH EVALUATION AND SEARCH FOR TEXT RECOGNITION}

In Section 2, we described the construction process of the csr-lattice shown in Figure 1(b) and (c). To obtain the optimal csr-lattice (optimal recognition path), we use the beam search strategy to search for optimal recognition path. Each path is evaluated by the path evaluation criterion proposed by Zhu et al. [5], which combines the scores of character recognition, linguistic context, and geometric features (character pattern sizes, inner gaps, single-character positions, pair-character positions, candidate segmentation points) with the weighting parameters estimated by the genetic algorithm.

Denote $X\left(x_{1}, \ldots, x_{m}\right)$ as successive candidate character patterns of one path, and every candidate character pattern $x_{i}$ is assigned candidate class $c_{i}$. Then $f(X, C)$ is the score of the path $(X, C)$ where $C=c_{1}, \ldots, c_{m}$. The path evaluation criterion is expressed as follows:

$$
f(X, C)=\sum_{i=1}^{m}\left\{\sum_{h=1}^{6}\left[\lambda_{h 1}+\lambda_{h 2}\left(k_{i}-1\right)\right] \log p_{h}+\lambda_{71} \log P\left(g_{j_{i}}\right) \mid S P+\lambda_{72} \sum_{j=j_{i}+1}^{j_{i}+k_{i}-1} \log P\left(g_{j} \mid N S P\right)\right\}+m \lambda
$$

where $P_{h}(h=1, \ldots, 6)$ stand for the probabilities of $P\left(c_{i} \mid c_{i-2} c_{i-1}\right), P\left(b_{i} \mid c_{i}\right), P\left(q_{i} \mid c_{i}\right), P\left(p^{u}{ }_{i} \mid c_{i}\right), P\left(p^{b}{ }_{i} \mid c_{i-1} c_{i}\right)$ and $P\left(x_{i} \mid c_{i}\right)$, respectively. $k_{i}$ is the number of primitive segments contained in the candidate character pattern $x_{i} . P\left(x_{i} \mid c_{i}\right)$ is estimated by the score of the on-line and off-line combined recognizers.

$P\left(c_{i} \mid c_{i-2} c_{i-1}\right)$ is the tri-gram linguistic context probability. In our system, we use smoothed tri-gram model by interpolation of uni-gram, bi-gram and tri-gram shown in Eq. (15), where $\lambda_{1}+\lambda_{2}+\lambda_{3}=1\left(\lambda_{1}=0.7, \lambda_{2}=0.2\right.$ and $\lambda_{3}=0.1$ in our experiment). The linguistic context model is trained by the year 1993 volume of the ASAHI newspaper and the year 2002 volume of the NIKKEI newspaper; the total size after pruning is 7.5 MB.

$$
P_{\text {smooth }}\left(c_{i} \mid c_{i-2} c_{i-1}\right)=\lambda_{1} \times P\left(c_{i} \mid c_{i-2} c_{i-1}\right)+\lambda_{2} \times P\left(c_{i} \mid c_{i-1}\right)+\lambda_{3} \times P\left(c_{i}\right)
$$

$b_{i}, q_{i}, p^{u}{ }_{i}$ and $p^{b}{ }_{i}$ are geometric feature vectors for character pattern sizes, inner gaps, single-character positions and pair-character positions, respectively; $b_{i}, p^{u}{ }_{i}$ and $p^{b}{ }_{i}$ are shown in Figure 4(a). $b_{i}$ is composed of height and width of the bounding box of the character pattern. $p^{u}{ }_{i}$ consists of two vertical distances from the vertical central line of the text line to top and bottom of bounding box. $p_{i}^{b}$ is also composed of two items. The two items are vertical distance between top edges of bounding boxes of two adjacent character patterns in text line and that of between bottom edges of bounding boxes. To obtain the inner gap of one character pattern, we obtain the statistical histograms of the pattern in horizontal and vertical direction by projecting the character pattern into the two directions; we divide each of histograms into three slices and the gap of slit in each slices normalized by the average size of text line that was denoted as acs are extracted as feature value of each items in feature vector $q_{i}$ as shown in Figure $4(\mathrm{~b}) . P\left(b_{i} \mid c_{i}\right)$, 
$P\left(q_{i} \mid c_{i}\right), P\left(p^{u}{ }_{i} \mid c_{i}\right)$ and $P\left(p^{b}{ }_{i} \mid c_{i-1} c_{i}\right)$ are evaluated by four quadratic discriminant function (QDF) classifiers trained by the Nakayosi database and they are together called geometric context. $g_{j}$ is the between-segment gap feature vector [21]. We approximate $P\left(g_{j_{i}} \mid S P\right)$ and $P\left(g_{j} \mid N S P\right)$ using a SVM classifier with a binary output trained by the feature data of sampled segmentation points labeled as SP (segmentation point) or NSP (not segmentation point) in the Kondate database.

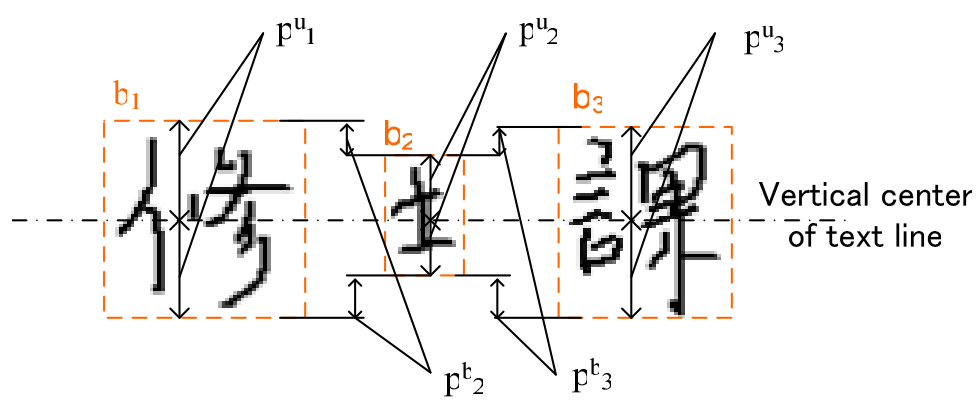

(a) $b_{i}, q_{i}, p^{u}{ }_{i}$ and $p^{b}{ }_{i}$

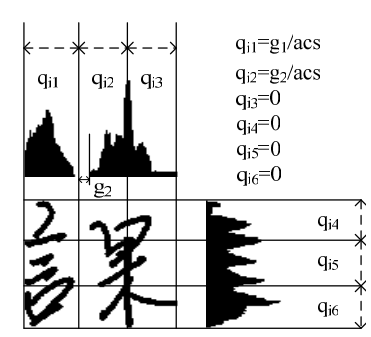

(b) Inner gap feature vector $q_{i}$

Figure 4: Geomeric feaure vectors used in text recognizer.

\section{TEXT RECOGNIZER AND EVALUATION}

\subsection{Experimental Data}

For training the weight parameters, we extracted horizontally written text lines from the database Kondate mentioned in Section 2. Among 100 people's patterns, we used 75 people's text lines to train the SVM classifier [19] for the evaluation of candidate segmentation and the weighting parameters of each component in path evaluation function. The 75 people's text lines contain 10,174 lines covering 1,106 character classes and the average length per line is about 10.23 characters. The on-line recognizer, off-line recognizer, and geometric scoring functions are trained by using the Nakayosi database.

To evaluate the performance of the text recognition system, we use the sentential part of each writer's file in the Kuchibue database (120 files) described in Section 2 as testing data. The sentential part of each writer's file includes 189 sentences, the longest having 204 characters, the shortest having 8 characters, and the average length being 53.7 characters. The experiments were implemented on an Intel(R) Xeon(R) CPU X5680 @ $3.33 \mathrm{GHz} 3.33 \mathrm{GHz}(2$ processers) with 48-GB memory.

\subsection{Experimental Results}

Table 3 (a) shows the accuracies of different text recognition systems that integrate linguistic context and geometric context on top of on-line and off-line recognition. We call them fully integrated recognizers in order to distinguish them from off-line recognizers, combined recognizers or partially integrated recognizers. Here the pair of parameters 
Table 3: Performance evaluation of different text recognizers

(a) Accuracies of differnt text recognizers (\%)

\begin{tabular}{|c|c|c|c|c|c|c|c|c|c|c|c|c|}
\hline \multicolumn{8}{|c|}{ Recognition accuracy $(\%)$ in CLD } & \multicolumn{5}{|c|}{ Recognition accuracy (\%) in SLD } \\
\hline $\mathrm{k} \backslash \mathrm{n}$ & 60 & 80 & 100 & 120 & 140 & 160 & 180 & $\mathrm{k} \backslash \mathrm{n}$ & 20 & 30 & 40 & 50 \\
\hline 10 & 98.26 & 98.34 & 98.31 & 98.33 & 98.33 & 98.32 & 98.29 & 2 & 97.82 & 97.98 & 98.10 & 98.17 \\
\hline 20 & 98.24 & 98.32 & 98.30 & 98.32 & 98.31 & 98.33 & 98.30 & 5 & 97.80 & 98.01 & 98.15 & $\mathbf{9 8 . 2 3}$ \\
\hline 30 & 98.19 & 98.29 & 98.27 & 98.29 & 98.32 & 98.30 & 98.27 & 10 & 97.82 & 98.01 & 98.14 & 98.23 \\
\hline 40 & 98.04 & 98.17 & 98.19 & 98.25 & 98.25 & 98.23 & 98.18 & 13 & 97.84 & 98.02 & 98.13 & 98.22 \\
\hline 50 & 97.76 & 97.96 & 98.01 & 98.09 & 98.11 & 98.09 & 98.01 & 16 & 97.86 & 98.03 & 98.12 & 98.21 \\
\hline 60 & 97.73 & 97.75 & 97.76 & 97.87 & 97.89 & 97.85 & 97.72 & 20 & 97.85 & 98.00 & 98.13 & 98.20 \\
\hline
\end{tabular}

(b) Memory-costs of different text recognziers (MB)

\begin{tabular}{|c|c|c|c|c|c|c|c|c|c|c|c|c|}
\hline \multicolumn{8}{|c|}{ Memory-cost (MB) in CLD } & \multicolumn{5}{|c|}{ Memory-cost (MB) in SLD } \\
\hline $\mathrm{k} \backslash \mathrm{n}$ & 60 & 80 & 100 & 120 & 140 & 160 & 180 & $\mathrm{k} \backslash \mathrm{n}$ & 20 & 30 & 40 & 50 \\
\hline 10 & 35.3 & 37.1 & 39.0 & 40.8 & 42.7 & 44.5 & 46.4 & 2 & 30.1 & 30.3 & 30.6 & 30.8 \\
\hline 20 & 40.5 & 44.0 & 47.6 & 51.1 & 54.7 & 58.2 & 61.8 & 5 & 30.6 & 31.1 & 31.6 & 32.1 \\
\hline 30 & 45.7 & 51.0 & 56.2 & 61.5 & 66.7 & 72.0 & 77.4 & 10 & 31.5 & 32.5 & 33.4 & 34.3 \\
\hline 40 & 51.0 & 57.9 & 64.8 & 71.8 & 78.7 & 85.7 & 92.6 & 13 & 32.1 & 33.3 & 34.5 & 35.7 \\
\hline 50 & 56.2 & 64.8 & 73.5 & 82.1 & 90.8 & 99.4 & 108.0 & 16 & 32.7 & 34.1 & 35.5 & 37.0 \\
\hline 60 & 61.5 & 71.8 & 82.1 & 92.4 & 103.0 & 113.1 & 123.4 & 20 & 29.5 & 35.2 & 36.2 & 38.7 \\
\hline
\end{tabular}

(c) Total average time-costs per character of different text recognizers (ms)

\begin{tabular}{|c|c|c|c|c|c|c|c|c|c|c|c|c|}
\hline \multicolumn{8}{|c|}{ Time-cost per character (ms) in CLD } & \multicolumn{5}{|c|}{ Time-cost per character (ms) in SLD } \\
\hline $\mathrm{k} \backslash \mathrm{n}$ & 60 & 80 & 100 & 120 & 140 & 160 & 180 & $\mathrm{k} \backslash \mathrm{n}$ & 20 & 30 & 40 & 50 \\
\hline 10 & 25.11 & 25.61 & 25.59 & 25.74 & 26.07 & 26.23 & 26.54 & 2 & 24.94 & 25.01 & 25.06 & 25.30 \\
\hline 20 & 25.63 & 25.76 & 25.82 & 26.07 & 26.43 & 26.62 & 26.94 & 5 & 24.78 & 25.03 & 25.02 & 25.32 \\
\hline 30 & 25.85 & 25.91 & 26.01 & 25.11 & 26.76 & 26.88 & 27.43 & 10 & 24.82 & 25.10 & 25.09 & 25.43 \\
\hline 40 & 25.91 & 26.20 & 26.32 & 26.58 & 27.05 & 27.71 & 27.83 & 13 & 25.04 & 24.88 & 25.19 & 24.52 \\
\hline 50 & 25.68 & 26.37 & 26.56 & 26.85 & 27.36 & 28.00 & 28.16 & 16 & 24.90 & 24.92 & 25.17 & 24.60 \\
\hline 60 & 25.84 & 26.53 & 26.80 & 27.14 & 27.70 & 25.48 & 28.59 & 20 & 24.95 & 24.97 & 25.18 & 24.59 \\
\hline
\end{tabular}

of the component off-line recognizer is also used to mark the fully integrated recognizers. Figure 5 shows the accuracy in a 3D surf graph. The memory-cost of the fully integrated recognizers is summation of those for coarse classification (10MB), on-line recognizer (12MB), off-line recognizer (variable), and linguistic context (7.5 MB), so it is $29.5 \mathrm{MB}$ plus memory-cost of the component off-line recognizer as shown in Table 3 (b).

Table 3 (a) and Figure 5 show (1) accuracy variation is relative flat, just $0.71 \%$ in CLD and $0.43 \%$ in SLD, and (2) surprisingly simple off-line recognizers (in SLD), which are weak on their own, are more accurate than those in CLD. If we select the off-line recognizer with 50 dimensions and 5 principal components (memory-cost 2.6 MB shown in Table 1 (b)) in the fully integrated recognizer, the total memory-cost of the system is reduced to $1 / 4$ (from 123MB to $32 \mathrm{MB}$ in Table 3 (b)) and the average time-cost per character recognition is reduced to nearly $4 / 5$ (from 0.1672 to $0.1349 \mathrm{~ms}$ in Table 2 (b)) while keeping almost the highest accuracy $98.23 \%$ (the best accuracy is $98.34 \%$ ) compared with the off-line recognizer of 180 dimensions and 60 principal components.

For character recognition alone, off-line recognizers with around 160 dimensions and 50 principal components have conventionally been used $[3,14]$. Compared with them, our selection of the off-line recognizer is surprisingly 


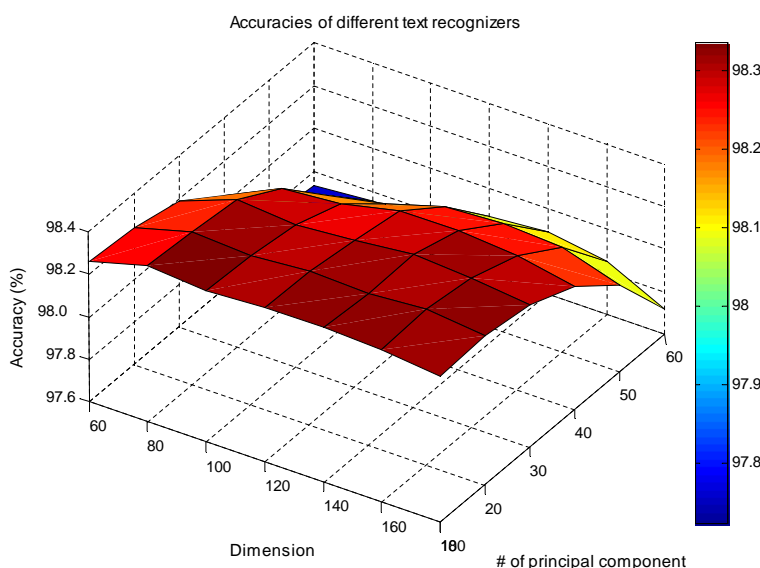

(a) Accuracy variation corresponding to CLD

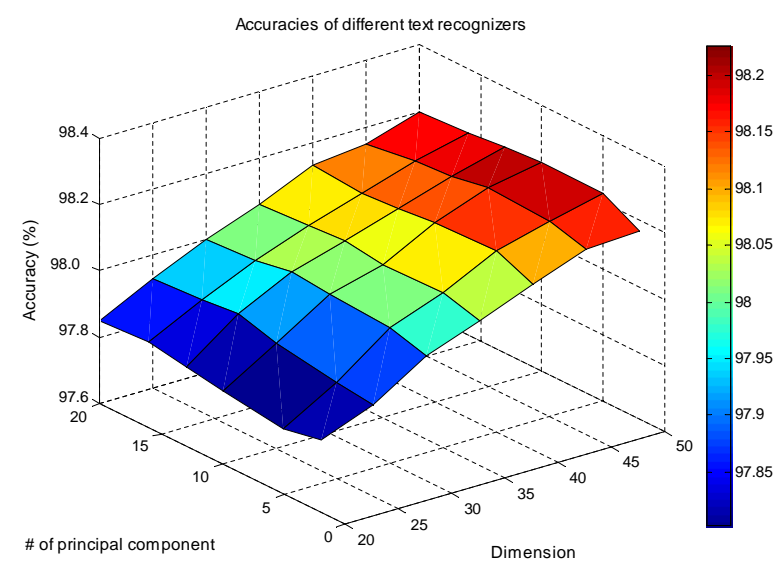

(b) Accuracy variation corresponding to SLD

Figure 5: 3D surf graph of accuracies by using different text recognizers.

small.

As for the total time-cost for the fully integrated recognizer, however, that of the combined recognizer (shown in Table 2 (b)) is rather small and that for the optimal path search takes a significant portion with the results shown in Table 3 (c). Therefore, the time-cost of the entire text recognition remains to be reduced.

Because a surprisingly simple off-line recognizer produces nearly the best result for text recognition, it seems natural to remove off-line recognizers from the combination. Without off-line recognizers, i.e., with only the on-line recognizer, however, the accuracy of the text recognition is $95.01 \%$ (3.3 point down from 98.34\%). Therefore, off-line recognition plays a significant role in text recognition.

\subsection{Experimental Result Analysis}

The accuracy variation between different off-line recognizers is large, ranging from $76.8 \%(\mathrm{n}=20, \mathrm{k}=2)$ to $90.7 \%$ $(\mathrm{n}=180, \mathrm{k}=60)$ as shown in Table 1 (a). Moreover, it is similar to that between different combined recognizers, which ranges from $91.10 \%(\mathrm{n}=20, \mathrm{k}=2)$ to $95.74 \%(\mathrm{n}=180, \mathrm{k}=40)$ as shown in Table $2(\mathrm{a})$, while that for fully integrated recognizers including linguistic and geometric context evaluation is rather small, ranging from $97.63 \%(n=20, k=2)$ to 98.34\% ( $\mathrm{n}=80, \mathrm{k}=10)$ as shown in Table 3 (a). The question is why such phenomenon occurs. Because the combined recognizer $(n=180, k=40)$ alone produces the best recognition accuracy as shown in Table $2(a)$, we will consider the reasons by the series of following comparative experiments with our selected combined $\operatorname{recognizer}(\mathrm{n}=50, \mathrm{k}=5)$ for the fully integrated recognizers.

\subsubsection{Impact of Accumulated Accuracy}

Although Figure 1(c) shows the candidate number $(\mathrm{CN})$ three for convenience, the candidate number for each candidate character pattern in our system is set as 20. Figure 6(a) shows the text recognition accuracy variation of 


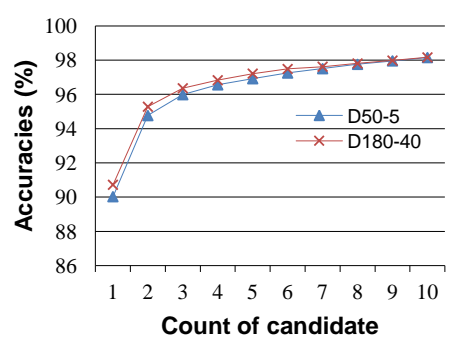

(a) Accuracy of text recognition with different (b) Accumulated accuracy of character recog- (c) $\mathrm{CN}$

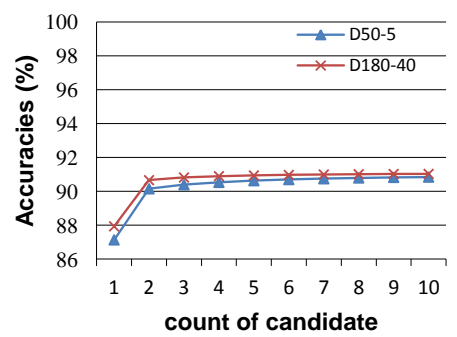

(c) Accuracy of text recognition without linguistic context

Figure 6: Impact of the accumulated accuracy of combined character recognizer, and linguistic context to text recognizer.

using the combined recognizer $(n=180, k=40)$ and our selected one $(n=50, k=5)$ for the fully integrated recognizer corresponding with different candidate numbers. Moreover, we present variation of the accumulated accuracy of the two combined recognizers for character recognition with different candidate numbers in Figure 6(b) (The testing database is the same as that in Section 5). According to Figure 6(b), the difference in performance between the two combined recognizers D180-40 and D50-5 becomes less and less as CN increases. When 10 is selected as the CN, the difference is about 0.06 points. Moreover, we can infer the difference in the two combined recognizers will become even less when $\mathrm{CN}$ is increased to 20 , which is selected for our fully integrated recognizer in accordance with the variation trend of accumulated accuracy with different $\mathrm{CN}$ shown in Figure 6(b). Therefore, the text recognition performance by fully integrated recognizers using these two combined recognizers also becomes very close $(98.18 \%$ and $98.23 \%$ ) since the accumulated recognition accuracy of the combined recognizer significantly contributes to the accuracy of the text recognition.

\subsubsection{Impact of the linguistic context information}

The linguistic context information is very important to the text recognition system. We show its importance to the fully integrated recognizers by removing the linguistic context component from the integrated frame for optimal path search described in Section 6. We do a similar experiment to that in Section 7.3.1, and present the variation of text recognition accuracy with a different $\mathrm{CN}$ as shown in Figure 6(c). Compared with the fully integrated recognizers, accuracy decreases 7 points (from about $98 \%$ in Figure 6(a) to about $91 \%$ in Figure 6(c) when 10 is selected as the $\mathrm{CN})$. Therefore, the linguistic context greatly contributes to increasing the accuracy.

\subsubsection{Impact of geometric context}

For further investigation, we remove geometric context from the path evaluation function described in Section 6 and generate text recognizers only including segmentation, character recognizer, and linguistic context. To distinguish them from fully integrated recognizers, we call them partially integrated recognizers. We also use the two combined recognizers D180-40 and D50-5 to recognize candidate character patterns in partially integrated recognizers. We use 
the same testing data as those in Section 7.1 to obtain the text recognition accuracy of the two partially integrated recognizers using the different combined recognizers D180-40 and D50-5, with results of 97.52\% and 97.62\%, respectively. This again shows that D50-5 is better than D180-40 in partially integrated recognizers. However, the difference in text recognition accuracy in two partially integrated recognizers is just 0.10 points, which is similar to the 0.04 -point difference $(98.18 \%$ and $98.23 \%)$ of the fully integrated recognizers. Moreover, the difference between the partially integrated recognizer and the fully integrated recognizer including D180-40 or D50-5 is small. i.e., 0.67 or 0.61 points, respectively, so the effect of geometric context is rather small.

From the above comparative experiments, accumulated accuracy of the combined recognizer, linguistic context and geometric context work together to increase the accuracy and decrease the weight of character pattern recognition among them. Even in a single recognizer, the phenomenon of "peaking out" occurs in a certain amount of features. In a system of multiple modules contributing to the recognition, a fewer features in each module may peak out.

\section{CONCLUSION}

In the paper, we have shown that a relatively weaker off-line recognizer with lower dimensions and fewer principal components brings in nearly the best accuracy while reducing memory and time cost drastically for text recognition when it is integrated with other components i.e., on-line character recognition, geometric context, and linguistic context. Even in a single recognizer, peaking out occurs in a certain amount of features. In a system of multiple modules contributing to the recognition, a fewer features in each module may peak out. Future work is reducing features in on-line recognition, geometric context evaluation, and linguistic context evaluation.

\section{ACKNOWLEDGMENTS}

This work has been supported by the R\&D fund for "development of pen \& paper based user interaction" under Japan Science and Technology Agency.

\section{References}

[1] H. Tanaka, K. Nakajima, K. Ishigaki, K. Akiyama and M. Nakagawa, "Hybrid Pen-input Character Recognition System Based on Integration of On-line and Off-line Recognition," Proc. of ICDAR '99, pp.209-212, 1999.

[2] F. Kimura et al., "Modified Quadratic Discriminant Functions and the Application to Chinese Character Recognition," IEEE Transactions On Pattern Analysis And Machine Intelligence, vol. PAMI-9, no.1, 1987.

[3] X.-D. Zhou,C.-L. Liu, Nagasaki and K. Marukawa, "Online handwritten Japanese Character String Recognition Incorporating Geometric Context," Proc. 9th ICDAR, Curitiba, Brazil, pp. 48-52, 2007.

[4] M. Nakagawa, B. Zhu and M. Onuma, "A model of On-line Handwritten Japanese Text Recognition Free from Line Direction and Writing Format Constraints," IEICE Trans. Inf. \& Syst., vol. E88-D, no.8, pp. 1815-1822, 2005.

[5] B. Zhu, X.-D. Zhou, C.-L. Liu and M. Nakagawa, ”A Robust Model for On-line Handwrittern Japanese Text Recognition,” IJDAR (2010) vol.13, no.1, PP. 121-131, 2010. 
[6] H. Oda, B. Zhu, J. Tokuno, M. Onuma, A. Kitadai and M. Nakagawa, ”Size Reduction of an On-line Handwritten Character Recognizer Combining On-line and Off-line Recognizers," IEICE Trans. Inf\&Syst., vol.J90-D, no.9, pp.2583-2594, 2007.

[7] M. Nakagawa and K. Matsumoto, "Collection of on-line handwritten Japanese character pattern databases and their analysis," International Journal on Document Analysis and Recognition, vol.7, no.1, pp. 69-81, 2004.

[8] Y. Katayama, S. Uchida and H. Sakoe, "HMM for On-Line Handwriting Recognition by Selective Use of Pen-Coordinate Feature and PenDirection Feature (in Japanese),” IEICE Trans. on Information and Systems, Vol. J91-D,no.8, pp. 2112-2120, 2008.

[9] S. Z. Li, Markov Random Field Modeling in Computer Vision, Springer-Verlag, New York, USA, 1995.

[10] J. Zeng and Z.-Q. Liu, ”Markov Random Fields for Handwritten Chinese Character Recognition,” Proc. of 8th Conference on Document Analysis and Recognition, Seoul, pp. 101-105, 2005.

[11] B. Zhu and M. Nakagawa, "A MRF Model with Parameter Optimization by CRF for On-line Recognition of Handwritten Japanese Characters," Proc. of Document Recognition and Retrieval XVIII (DRR), USA, 2011.

[12] U. Ramer, "An Iterative Procedure for the Polygonal Approximation of Plan Closed Curves," Computer Graphics and Image Processing, vol.1, no.1, pp.244-256, 1972.

[13] G. David Forney, Jr., ’The Viterbi Algorithm,” Proc. of the IEEE, vol.61, no.3, pp.268-278, 1973.

[14] C.-L. Liu and X.-D. Zhou, "Online Japanese Character Recognition Using Trajectory-based Normalization and Direction Feature Extraction," Proc. of 10th International Workshop on Frontiers in Handwriting Recognition,” pp.217-222, 2006.

[15] C.-L. Liu and K. Marukawa, "Pseudo two-dimensional Shape Normalization Methods for Handwritten Chinese Character Recognition," Pattern Recognition, vol.38, no.12, pp. 2242-2255, 2005.

[16] R.V.D. Heiden, F.C.A. Gren, ”The Box-Cox Metric for Nearest Neighbor Classification Improvement,” Pattern Recognition, vol.30, no.2, pp. 273-279, 1997.

[17] Peter N. Belhumeur, "Eigenfaces vs Fisherfaces Recognition Using Class Specific Linear Projection,” IEEE Transactions on Pattern Analysis and Machine Intelligence, vol.19, no.7, 1997.

[18] R. P. W. Duin and R.Haeb-Umbach, ”Multiclass Linear Dimension Reduction by Weighted Pairwise Fisher Criteria,” IEEE Transactions On Pattern Analysis And Machine Intelligence, vol.23, no.7, 2001.

[19] J. Kittler, M. Hatef, R. Duin, and J. Matas, ”On combining Classifiers," IEEE Transactions On Pattern Analysis And Machine Intelligence, vol.20, no.3, pp. 123-140,1998.

[20] B.-H. Juang, W. Chou and C.-H. Lee, ’Minimum classification error rate methods for speech recognition,” IEEE Trans. on Speech and Audio Processing, vol. 5, pp. 257-265, 1997.

[21] B. Zhu and M. Nakagawa, ”Segmention of on-line Freely Written Japanese Text Using SVM for Improving Text Recognition,” IEICE Trans. Inf\&Syst., vol. E91-D, no.1, pp. 105-113, 2008. 
Graphical Abstract

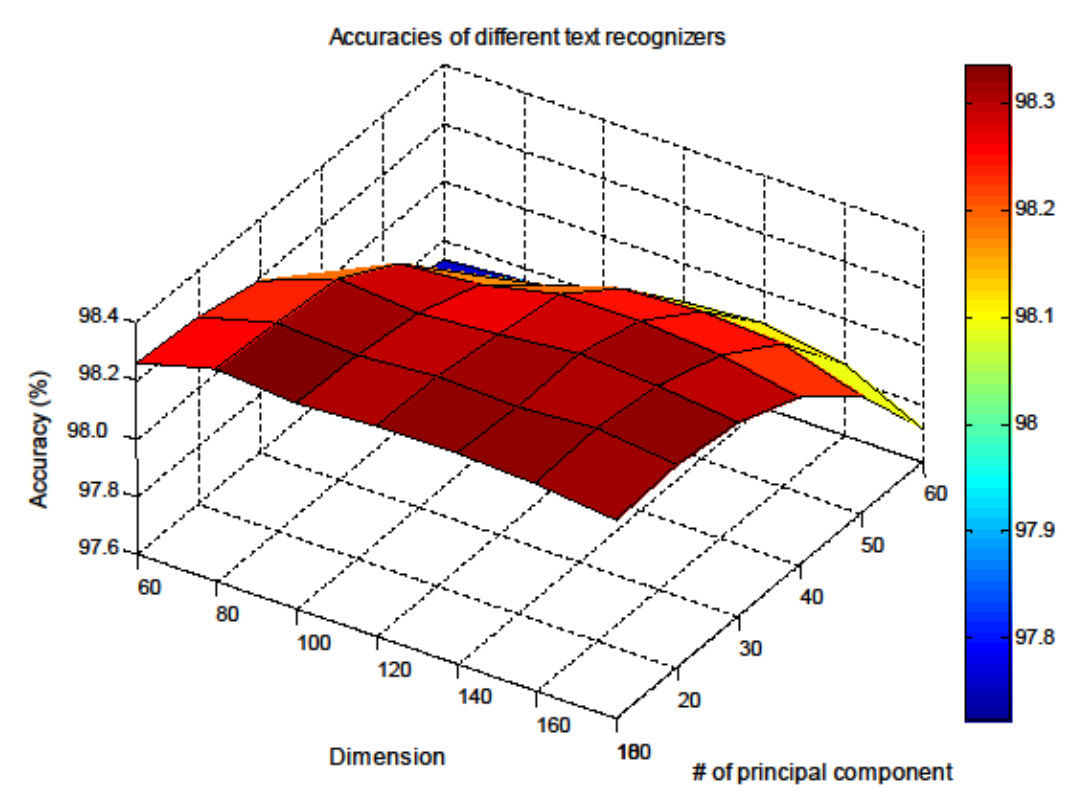

(a) 3D surf graph of text recognition accuracies corresponding to CLD part in Table 3,

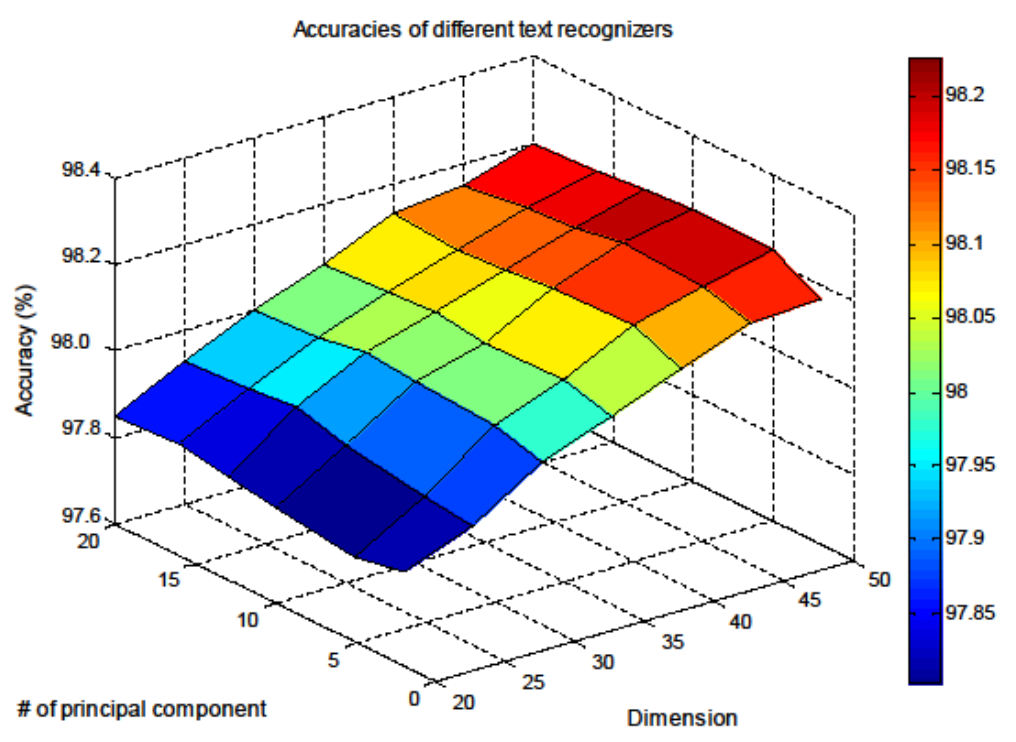

(b) 3D surf graph of text recognition accuracies corresponding to SLD part in Table 3.

Fig. 5 3D surf graph of text recognition accuracies by using different text recognizers 